\title{
Concurrent adaptation to opposing visual displacements during an alternating movement
}

\author{
J. M. Galea · R. C. Miall
}

Received: 28 November 2005/ Accepted: 2 June 2006/Published online: 12 July 2006

(C) Springer-Verlag 2006

\begin{abstract}
It has been suggested that, during tasks in which subjects are exposed to a visual rotation of cursor feedback, alternating bimanual adaptation to opposing rotations is as rapid as unimanual adaptation to a single rotation (Bock et al. in Exp Brain Res 162:513-519, 2005). However, that experiment did not test strict alternation of the limbs but short alternate blocks of trials. We have therefore tested adaptation under alternate left/right hand movement with opposing rotations. It was clear that the left and right hand, within the alternating conditions, learnt to adapt to the opposing displacements at a similar rate suggesting that two adaptive states were formed concurrently. We suggest that the separate limbs are used as contextual cues to switch between the relevant adaptive states. However, we found that during online correction the alternating conditions had a significantly slower rate of adaptation in comparison to the unimanual conditions. Control conditions indicate that the results are not directly due the alternation between limbs or to the constant switching of vision between the two eyes. The negative interference may originate from the requirement to dissociate the visual information of these two alternating displacements to allow online control of the two arms.
\end{abstract}

Keywords Physiological adaptation $\cdot$ Motor learning · Motor control $\cdot$ Interference $\cdot$ Kinematics

Support contributed by: Wellcome Trust \& EPSRC studentship.

J. M. Galea $(\bowtie) \cdot$ R. C. Miall

Behavioural Brain Science, School of Psychology,

University of Birmingham, Birmingham, UK

e-mail: jmg111@bham.ac.uk

\section{Introduction}

In every day life we produce a multitude of movements which involve a close interaction between two or more limbs ranging from opening a jar to driving a car. Even though there has been much research investigating these forms of movement it still remains unclear to what degree the limbs are controlled independently or concurrently. A major question is whether the most efficient way to control the separate upper limbs is through combining their control mechanisms at a high neural level or for their control to remain independent from one another. Wang and Sainburg (2003) have shown that, during a unimanual movement, learning a visuomotor rotation with one arm did not interfere with learning the opposite visuomotor rotation with the other arm. They concluded that the neural processes of each arm are independent. Transfer of learning is not obligatory but, when beneficial, they suggest a link can be made between the control mechanisms.

Hence there is a possibility that the two control systems remain largely independent but converge functionally at various areas in the brain. Evidence for this convergence is found in a number of different studies. Performing two different tasks simultaneously often results in mutual interference but dissociation can occur with training, which suggests that learning is required to separate the incoming information within these various areas (Wenderoth et al. 2003). Eliassen et al. (2000) have shown that callosal transmission affects the degree of bilateral synchrony within simple bimanual movements implying that the corpus callosum is integral in synchronizing the movement. Wenderoth et al. (2004) believe directional interference emerges primarily within superior parietal, int- 
raparietal and dorsal premotor areas of the right hemisphere. In particular they hypothesize that interference occurs when computational resources in the parietal areas are insufficient to code two incompatible movement directions independently from each other. In addition, Johansson et al. (2006) believe that when performing a bimanual task there is high degree of interaction between the two limbs. They showed that subjects are able to choose the hand with the greatest natural spatial mapping between actions and goals as the primary actor, irrespective of the preferred hand. This involved a high degree of flexibility and communication between the limbs as their roles were interdependent on one another. It implies that bimanual performance involves two independent actions evolving into a meaningful gestalt which can then be adapted to perform optimally in a given environment (Swinnen and Wenderoth 2004).

In contrast, no interference has been found when subjects learn two novel opposing visual rotations during alternating pointing tasks. Mikaelian and Malatesta (1974) and Prablanc et al. (1975) used prismatic displacement, exposing subjects to a visual displacement for one arm and then the opposite displacement for the other arm. They found that both arms adapted to the specific displacement and suggested independent neural mechanisms for the two limbs. Bock et al. (2005) extended this finding using a visual distortion produced via a computer screen. They found the time course of adaptation was similar for both hands and the magnitude of adaptation was comparable to unimanual data. They concluded that the two adaptive states were formed concurrently and without interference or facilitation.

However, an important criticism of these three studies is that the subjects learnt the opposing displacements during epochs of unimanual movement, which intermittently changed from side to side on a time scale ranging from $20 \mathrm{~s}$ (Bock et al. 2005) up to 3 min (Prablanc et al. 1975). Even with the 20-s scenario, the subject produced up to 15 movements before swapping arms. Hence there is considerable opportunity for unilateral adaptation within each of these epochs.

Another important limitation in Bock et al. (2005) is their comparison of unimanual and alternating adaptation using data from two separate studies. As a consequence they were unable to compare the full time course of adaptation, and could only compare the magnitudes of adaptation after a given time. They compared the point at which the adaptation curves reached the mean absolute error and found that these were not significantly different.
The aim of this study was to investigate if interference occurred when the visual displacement and limb involved changed after every movement in an alternating fashion. The comparison of unimanual and alternating conditions would take place over the full time course of adaptation using performance measures indicating both the feedforward and online correction components of the movement. We have compared alternating conditions with visually opposing and visually consistent prismatic displacements. In addition, control conditions would identify whether the alternation of the limbs and vision were factors which influenced adaptation rate. Through these series of conditions our aim was to explore in more detail the process of dual adaptation within an alternating task. Of interest was whether we could replicate Bock et al.'s (2005) results when alternation occurred after every movement. In particular a lack of interference in the alternating condition would suggest that the control systems of the two upper limbs were independent of each other throughout the adaptation process.

\section{Materials and methods}

\section{Participants}

Sixty healthy subjects (17 males, 43 females) with a mean age of 20 (S.D. 3.3 years) took part. They were all right-handed and had normal or corrected to normal vision. The subjects were recruited from the university community and received course credits to participate. All subjects provided informed consent. Right handedness was assessed using the 10-item version of the Edinburgh inventory (Oldfield 1971). This study was approved by the school ethics committee and therefore was performed in accordance with ethical standards laid down in the 1964 Declaration of Helsinki.

\section{Materials}

Subjects sat as close as possible to a wooden table. In front of each hand was a start button $7 \mathrm{~mm}$ in diameter, approximately $10 \mathrm{~cm}$ in front of each shoulder. Two additional $7 \mathrm{~mm}$ targets were positioned $35 \mathrm{~cm}$ away, in the saggital plane. The targets were $32 \mathrm{~cm}$ apart (Fig. 1), each placed centrally on a $20 \mathrm{~cm} \times 20 \mathrm{~cm}$ response plate.

The start and target switches were coupled to goggles with liquid crystal shutters (PLATO, Translucent Technology, Toronto, Canada). There were two sets of 


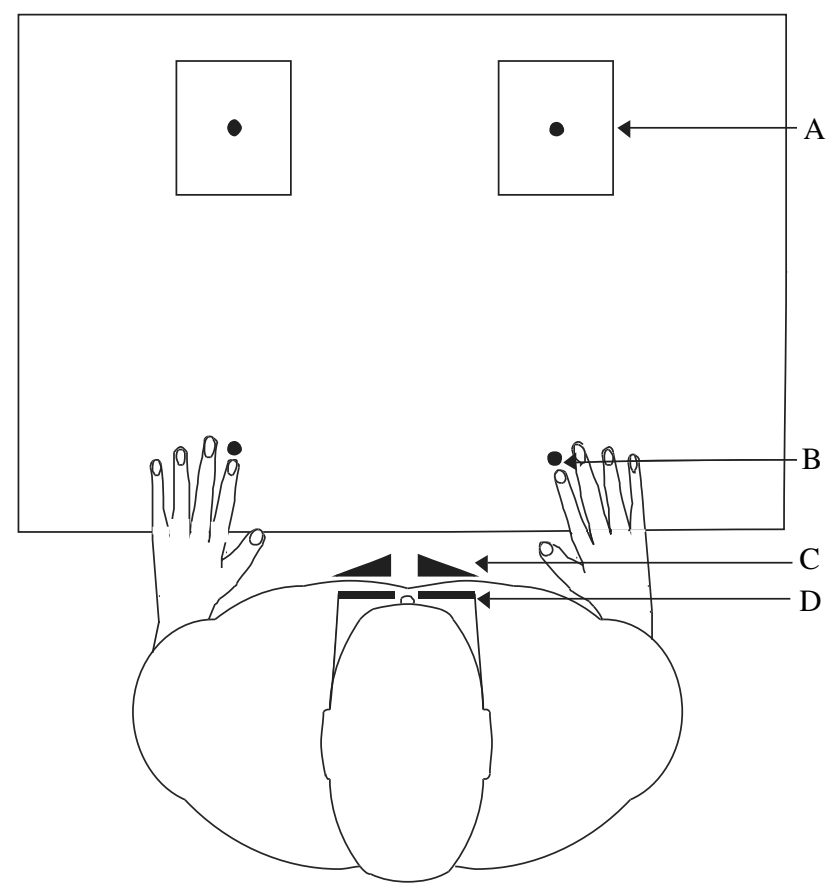

Fig. 1 Experimental set-up of the targets (a), start buttons (b) and subjects position. During adaptation, the shutters (d) blocked vision through the non-ipsilateral lens (c). Thus when moving the right hand, subjects had online vision via the right lens and vice-versa

goggles, one set were normal while the other set had self-adhesive prismatic Fresnel lenses (3M, Press-on, St Paul, MN, USA) attached to each lens. For the alternating opposing $(\mathrm{AO})$ condition the left lens caused a visual displacement of $11.4^{\circ}$ in an anti-clockwise direction, while the right lens caused a similar displacement in a clockwise direction. For the alternating consistent (AC) condition both lenses caused a visual displacement in a clockwise direction.

The movement of the index fingers was sampled using the Polhemus tracking system (Colchester, VT, USA) at a frequency of $60 \mathrm{~Hz}$.

\section{Procedure}

The subjects were informed that their main objective was to hit the targets as accurately as possible using their index fingers. Subjects were randomly allocated to one of six groups, which all followed a protocol of three sessions (Table 1). The pre- and post-adaptation sessions involved both hands moving simultaneously to and from the targets with the subjects only receiving vision when pressing the starting position and when hitting the target boards but not during the movement. This was to test whether the adaptive states could be concurrently and independently engaged in motor 
control. Vision was restricted to endpoint information as de-adaptation occurred within the first movement with unrestricted vision.

The subjects were told that the movement should be continuous between the starting buttons and targets, and at each end they should press down causing the switches to close. A metronome was used to ensure a similar movement speed across all subjects and conditions ( $1 \mathrm{~s}$ per movement) however the participants were reminded to focus on their movement accuracy and not speed.

The six groups differed in terms of the prism adaptation session that they experienced. The alternating adaptation conditions (AO and $\mathrm{AC}$ ) involved the subject beginning with both fingers pressing down on the start buttons and then either the left or right hand moved to the target situated on that side, whilst the other hand remained on the button. For half the subjects their right hand moved first while for the other half their left hand moved first. The release of the leftor right-sided start button resulted in left or right eye monocular vision, respectively during the movement to the target. Once the hand moving had travelled to the target and back to the starting position, pressing the switch, the other hand could begin its movement. The left and right hand moved in strict alternation. This ensured each hand only received vision from one eye and thus the hands learnt separate visual displacements. The AO condition involved the left hand adapting to a counter clockwise displacement and the right hand adapting to a clockwise displacement. The $\mathrm{AC}$ condition involved both the left and right hand adapting to a clockwise displacement. Thus the AO condition involved opposing visual displacements and the AC condition involved visually similar displacements.

The unimanual adaptation conditions involved only the left or right hand moving, respectively, whilst the other hand remained on the starting button, thus still only allowing monocular vision via the ipsilateral lens.

Two control conditions were used. Control 1 involved an alternating movement of both hands; however only the right hand movement involved adaptation to a visual displacement. This was to ensure that if interference did occur between the limbs it was as a result of learning to adapt to two visual displacements and not the alternation between the limbs. Control 2 involved a unimanual right handed movement however, the subject's unilateral vision alternated after every movement. This was to ensure that any change in adaptation rate during the alternating conditions was not due to the subjects having to switch between left eye and right eye, a process which might withdraw computational resources from the adaptation process.

Analysis

Two measures of task performance were calculated; hand-path direction error at peak tangential hand velocity (angle) and the final position error of the index finger for each pointing movement towards the distal target (error). We assumed that the angle measurement would represent the pre-planned aspect of the movement, whilst the error measurement would also reflect online correction. Hand-path direction error was calculated as the angular difference between the starting marker and the point of maximal hand velocity. A positive value indicated an initial displacement to the right and a negative value to the left of the line joining the starting marker and targets. Final position error was calculated as the horizontal error of the index finger, with relation to the target, as it made contact with the target board.

For statistical analysis epochs were created averaging error across three successive movements. Therefore, for the pre- and post-adaptation sessions there were 5 epochs, and the prism adaptation session consisted of 30 epochs (90 movements). The five preadaptation epochs were averaged to produce a baseline error for each subject. This was the then subtracted from each of their post-adaptation epochs leaving the error above baseline.

Within the analysis comparisons were performed for both measures of task performance. Analysis of variance with repeated measures ( 2 hands $\times 30$ epochs) were used to compare the left and right hand within the alternating conditions (AO and AC). These two conditions were then compared separately for the left and right hands ( 2 conditions $\times 30$ epochs). In order to measure the performance within these conditions in comparison to unimanual movement, separate ANOVAs compared each hand with the corresponding unimanual left or right hand condition (2 conditions $\times 30$ epochs). The two control conditions were compared with the unimanual right condition ( 3 conditions $\times 30$ epochs). When significant interactions were found between conditions and epochs, independent $t$-tests were performed on the 1st and 30th epoch comparing the performance between each condition. This aimed to test initial performance error and the extent of final adaptation, respectively.

Finally, for each group, an exponential learning model was fitted to the average epoch data for the prism adaptation session: 
$E(i)=\alpha^{\prime}+\rho \mathrm{e}^{-(i / \tau)}$

In the model, $E$ is the average group data from epoch $i$ relative to the start of the session. $\alpha^{\prime}$ is a scalar offset representing a subject's performance learning plateau, $\rho$ is the gain and $\tau$ represents the time constant of adaptation. A comparison of the left and right hand across conditions were performed by calculating the standard error of the $\tau$ value. The standard error indicated the possible variance of these values.

\section{Results}

\section{Summary}

Figure 2 shows the final position error (error) for each trial across the three sessions prior to averaging across
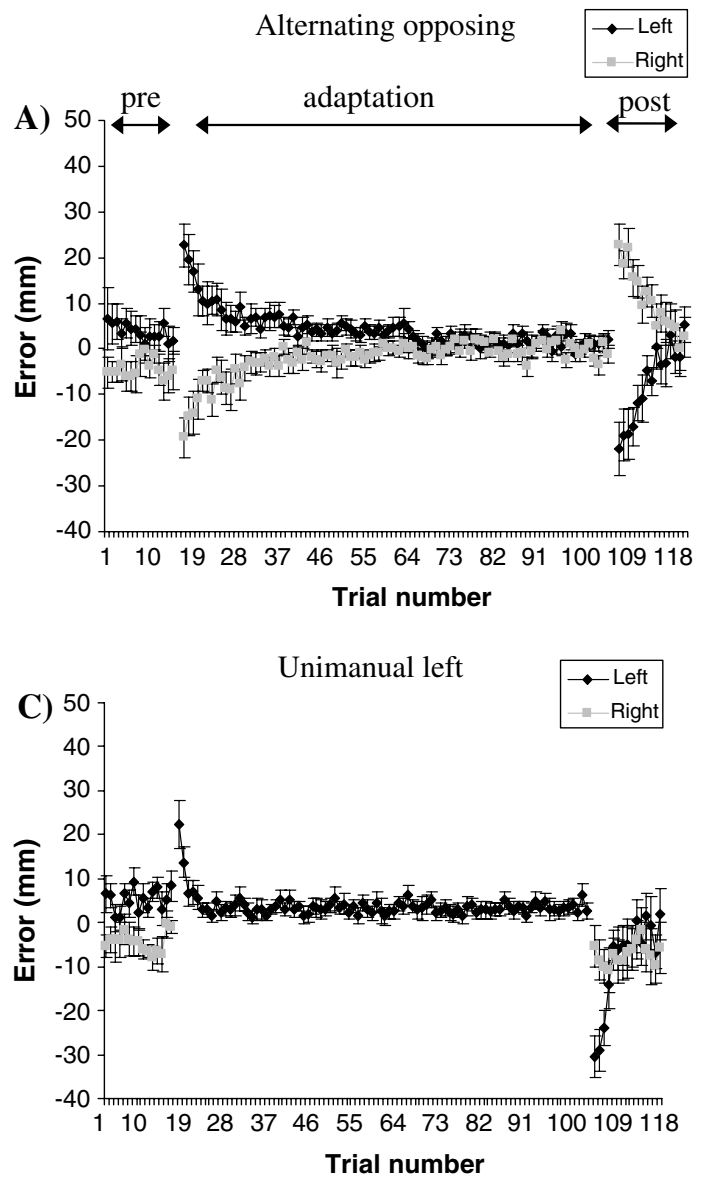

Fig. 2 Average final position error (error) within each condition. Trials $0-15=$ pre-adaptation session, 17-107=prism adaptation session, 108-122=post-adaptation session. Each marker represents the mean final position error $( \pm$ S.E. $)$, across subjects, for a given trial. Even prior to epoch formation it is clear that when exposed to a prismatic displacement the unimanual conditions (c and d) adapt at a quicker rate in comparison to the alternating epoch and removing baseline errors in each condition. The pre-adaptation session is characterized by relatively accurate performance. The prism adaptation session shows an initial large error due to the prismatic displacement; however over subsequent movements adaptation is seen as the error values return back to baseline. Within the post-adaptation session an aftereffect is seen as an error in the opposite direction to the initial displacement, with this fading over subsequent trials under the return to normal vision. Similar performance is seen within the hand-path directional error (angle) (Fig. 3).

Significant differences between the conditions were found for the error data. For angle data within each condition a significant main effect of epoch was found $(F(29,493) \geq 2.983, P \leq 0.0005)$; however the main effect of condition and the interaction between condition and epoch were found to be non-significant. This
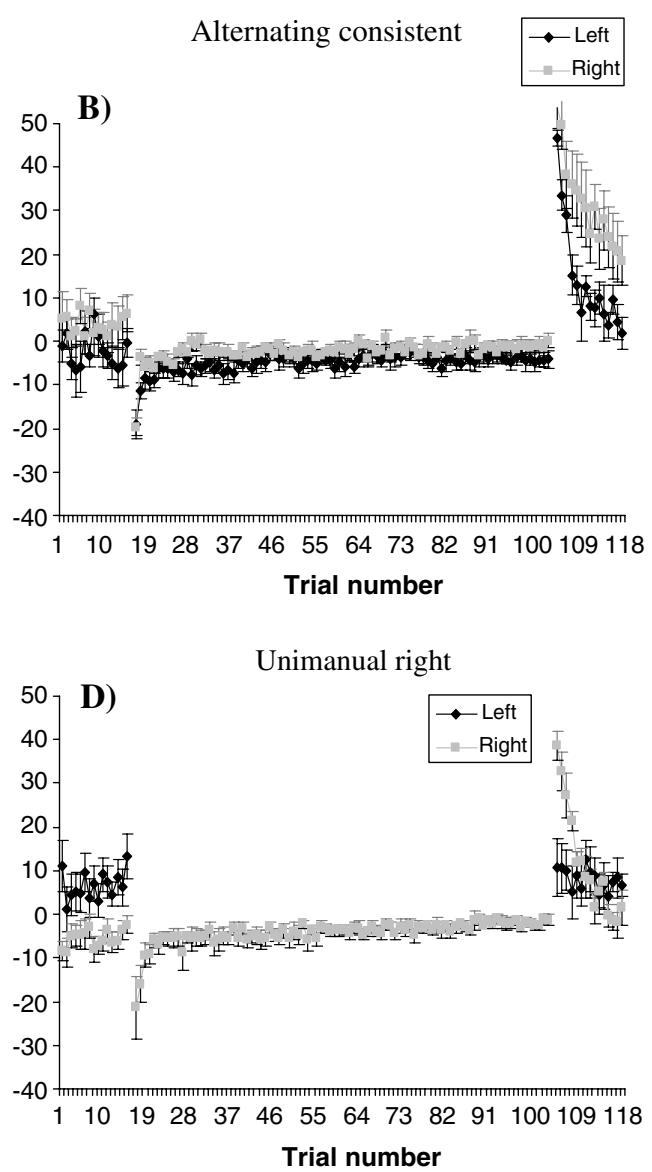

opposing condition (a). However the alternating consistent condition (b) has a similar adaptation pattern to the unimanual conditions. Within the post-adaptation session the unimanual conditions (c and d) and the alternating consistent condition (b) produce larger aftereffects which exceed the size of initial displacement 

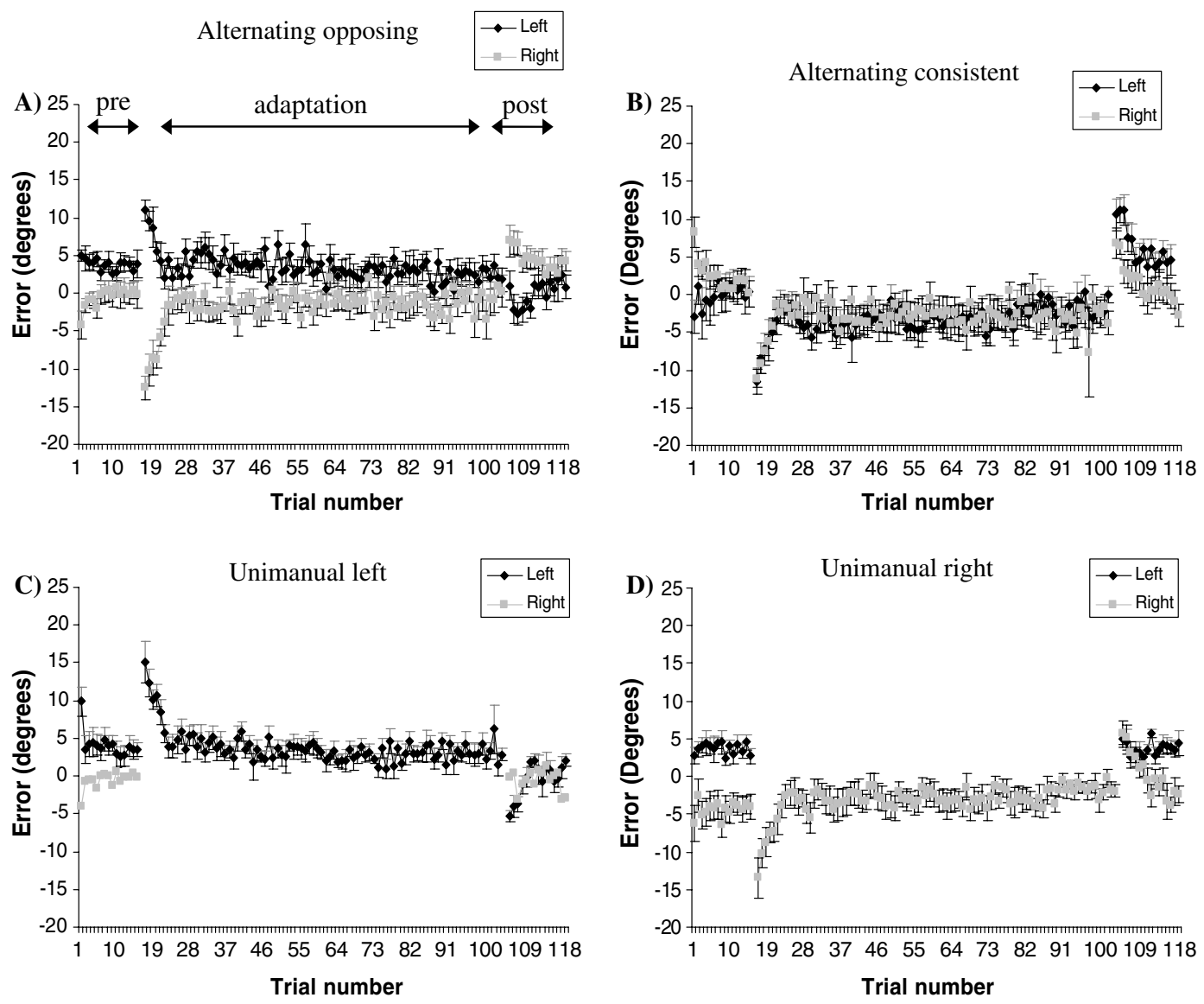

Fig. 3 Average hand-path direction error at peak tangential hand velocity (angle) within each condition. Trials $0-15=$ preadaptation session, 17-107=prism adaptation session, 108-122=post-adaptation session. Each marker represents the

was supported by the $\tau$ values shown in Fig. $6 \mathrm{~b}$ which indicates a similar rate of adaptation across conditions. As a result, unless specifically mentioned, only error data is subsequently presented.

Comparison of the left and right hands within the alternating and unimanual conditions

The improvement seen in the prism adaptation session was validated by within-subject ANOVAs (hand $\times \mathrm{e}$ pochs) which separately compared the left and right hand within the alternating conditions across epochs. For both the angle and error data the only significant effect was epochs $(F(29,261) \geq 1.879, P \leq 0.006)$. For the unimanual conditions a mixed ANOVA (condition $\times$ epochs) compared the adapted hand within each condition. For the angle and error data the only significant effect was epochs $(F(29,522) \geq 6.695, P \leq 0.0005)$.

This suggests that for all conditions and for both movement parameters significant adaptation to the

mean final position error $( \pm$ S.E. $)$, across subjects, for a given trial. In comparison to when looking at the error data this data suggests that the performance between the conditions is more similar

prismatic displacement took place. As no significant effect of hand or condition was found it indicates that the initial displacement and final level of adaptation was statistically similar for the left and right arm in either a unimanual or an alternating situation. With respect to the alternating conditions these results imply that independent adaptive states were formed concurrently in response to the specific displacement the hand was exposed to.

Comparison of the AO condition with the unimanual conditions (UL and UR)

If similar adaptation was seen in the alternating and unimanual conditions, it would suggest that the two alternating adaptive states were formed without any interference from one another. To investigate this further, direct comparisons were performed for each hand, comparing the alternating conditions (AO and $\mathrm{AC}$ ) and the appropriate unimanual conditions (UL or 
UR). Mixed ANOVAs (condition $\times$ epochs) compared the left hand within AO vs UL and separately the right hand within $\mathrm{AO}$ vs UR.

While the main effect of condition was not significant, the interaction between epochs and conditions was significant $(F(29,522) \geq 4.807, P \leq 0.039)$ for both comparisons. This suggests that there was a time dependent variation between the unimanual conditions and the AO condition. Independent $t$-tests compared the conditions within the 1st and 30th epochs. For both the left and right hand comparisons there no significant difference between the AO condition and unimanual condition at epoch 1 or 30 .

The exponential learning model (Eq. 1), applied to each condition, produced $\tau$ values which suggested that adaptation in the two unimanual conditions was quicker than in the alternating conditions. The left and right hand within the AO condition had $\tau$ values of 0.26 (S.E. 0.04) and 0.31 (S.E. 0.036), respectively while the unimanual conditions had $\tau$ values of 0.65 (S.E. 0.13) for the left and 1.85 (S.E. 0.41) for the right hand

A) AO.left

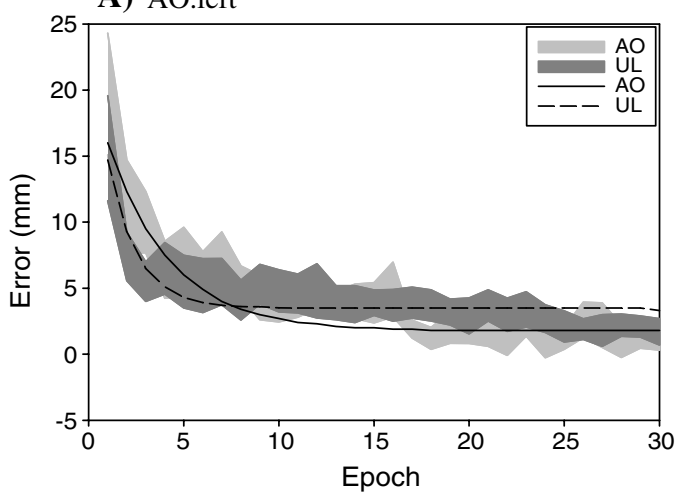

C) AC.left

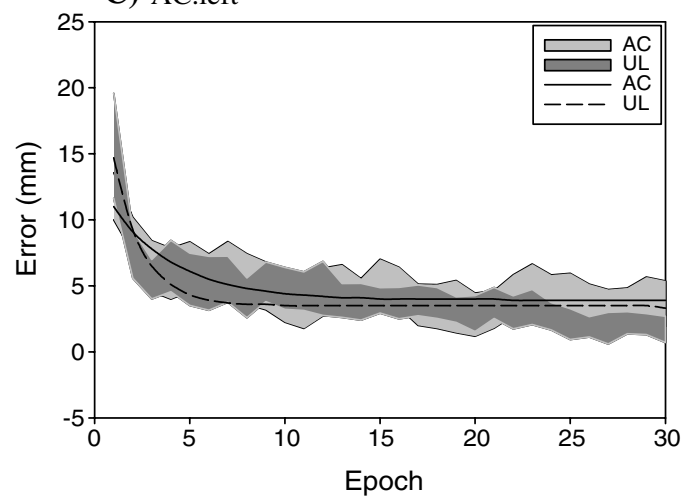

Fig. 4 a $A O$ vs UL, b AO vs UR. It is clear that the two hands within the AO condition adapted to their specific visual displacement. However in both instances subjects took longer to reach the plateau stage of adaptation in comparison to the corresponding unimanual condition. c AC vs UL, d AC vs UR.
(Fig. 6a). Fig. 4a, b applies the exponential learning curves onto the average epoch data. The larger $\tau$ value is reflected by the unimanual conditions reaching the plateau stage of adaptation at an earlier epoch.

Comparison of the AC condition with the unimanual conditions (UL and UR)

The AC condition involving visually similar prismatic visual displacements was compared with the appropriate unimanual conditions (UR or UL). While the main effect of condition was not significant, the interaction between epoch and condition was significant $(F(29,493)=2.554, P \leq 0.0005)$ for the right hand comparison. Independent $t$-tests compared the performance of the right hand within each condition in epochs 1 and 30. A significant difference was found at epoch $1(t=3.351, d f=18, P \leq 0.002)$ however not at epoch 30 . Figure $4 \mathrm{~d}$ shows that the right arm within the AC condition had a smaller initial error in comparison to the unimanual condition.

B) AO.right
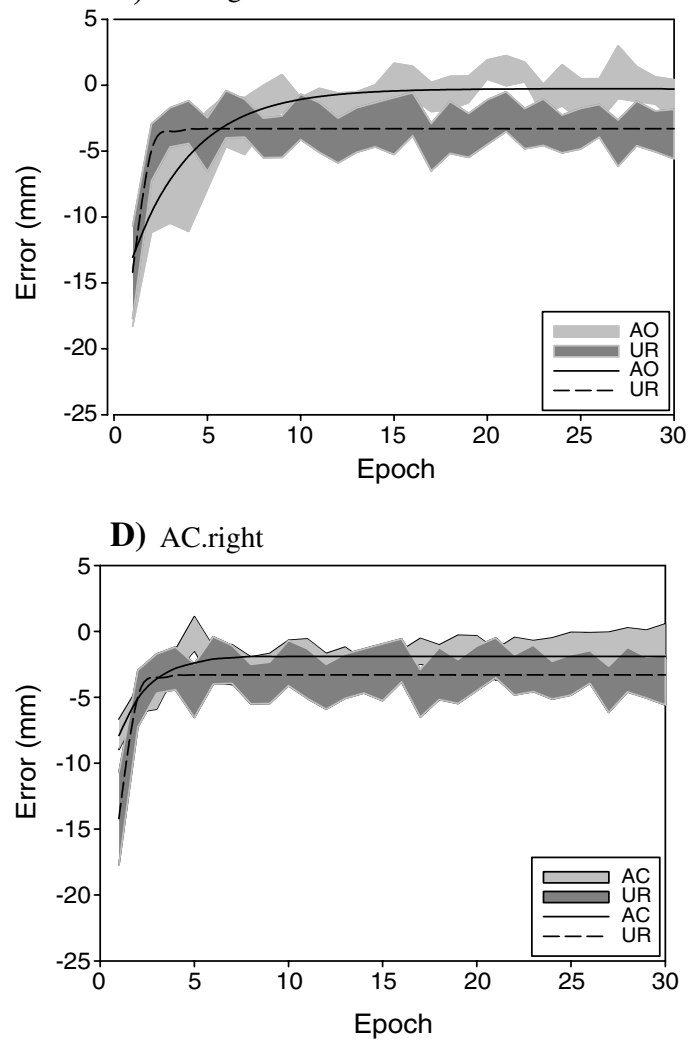

The unimanual conditions reach the plateau level of performance at an earlier epoch however the differences between the $\mathrm{AC}$ and unimanual conditions are not as clear as in the comparison between $\mathrm{AO}$ vs unimanual 
The exponential learning models applied (Eq. 1) indicate that the unimanual conditions had a faster rate of adaptation than the AC condition. The left and right hand within the AC condition had $\tau$ values of 0.3 (S.E. 0.05 ) and 0.625 (S.E. 0.151), respectively while the unimanual conditions had $\tau$ values of 0.65 (S.E. 0.13) for the left and 1.85 (S.E. 0.41) for the right hand (Fig. 6a). Figure 4c, d applies the exponential learning curves onto the average epoch data. Figure $4 c$, d shows the more rapid return to asymptotic performance for the unimanual conditions compared to the appropriate hand within condition AC.

Comparison of the alternating conditions (AO and $\mathrm{AC}$ )

The AO condition involved visually opposing prismatic visual displacements while the AC condition involved visually similar. There was a main effect of epoch $(F(29,493) \geq 7.418, P \leq 0.0005)$ however the main effect of condition was not significant. For the left and right hand comparisons the interaction between condition and epoch was significant $(F(29,493) \geq 1.760$, $P \leq 0.009)$. Independent $t$-tests were performed on epochs 1 and 30. For the left hand no significant differences were found however; for the right hand a significant difference was found at epoch $1(t=1.883$, $d f=18, P \leq 0.038)$. Fig. $5 \mathrm{~b}$ shows that the right hand within the $\mathrm{AO}$ condition produced a larger initial error in comparison to the right hand in the AC condition.

The learning models applied (Eq. 1) suggest that the left hand within both conditions produced similar rates of adaptation $(\mathrm{AO}=0.26$, S.E. 0.03, $\mathrm{AC}=0.3$, S.E. 0.05 ) (Fig. 6a). The slightly larger $\tau$ value produced within the AC condition may explain the interaction

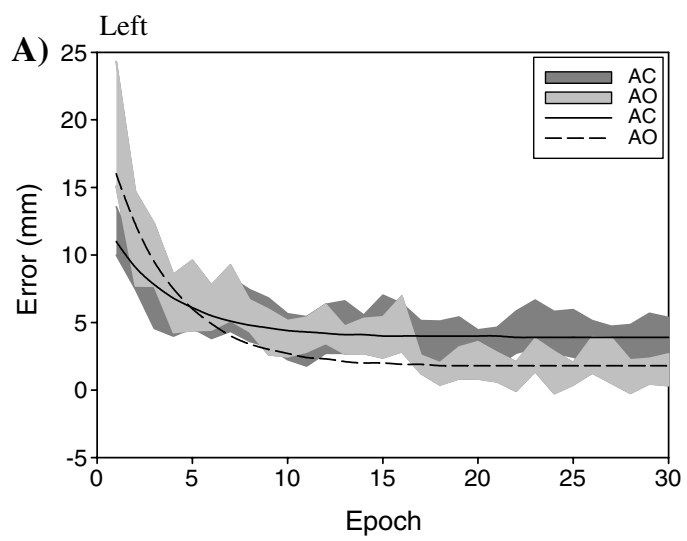

Fig. 5 A comparison of the left (a) and right (b) hand within the two alternating conditions ( $\mathrm{AC}$ and $\mathrm{AO}$ ). Adaptation within the AC condition occurred at a slightly faster rate with this being shown by the smaller initial error and faster return to asymptotic found and implies that the left hand within this condition adapted slightly faster than in the AO condition. For the right hand the $\tau$ values were significantly different $(\mathrm{AO}=0.3$, S.E. 0.03; $\mathrm{AC}=0.5$, S.E. 0.08). Figure 5 shows the more rapid return to asymptotic performance for both the left and right hand in the AC condition, compared to AO.

\section{Control conditions}

Control 1 involved an alternating movement with normal vision for left arm trials, but a clockwise visual displacement for right hand trials. The aim was to test if the slowed adaptation rate within $\mathrm{AO}$ and $\mathrm{AC}$ compared to unimanual conditions was due to the alternation of the movement or whether interference only occurred during dual adaptation. It was hypothesized that if the alternation of the two hands was directly causing the slowed adaptation rate within the alternating conditions then differences would be seen between control 1 and condition UR. A main effect of epoch was found $(F(29,522)=14.771, P \leq 0.0005)$, however the main effect of condition and the interaction between epoch and condition were both non-significant. However the learning models (Eq. 1) applied suggest that within condition UR a faster rate of adaptation ( $\tau=1.85$, S.E. 0.41$)$ was occurring in comparison to control $1(\tau=1.01$, S.E. 0.131).

There was also a possibility that the alternation of vision from left to right eye during the alternating tasks was a factor in causing the slowed adaptation rate. Control 2 involved a unimanual movement with the right arm. Subjects had to adapt to a clockwise visual displacement whilst their monocular vision alternated after every movement. It was hypothesized that if the

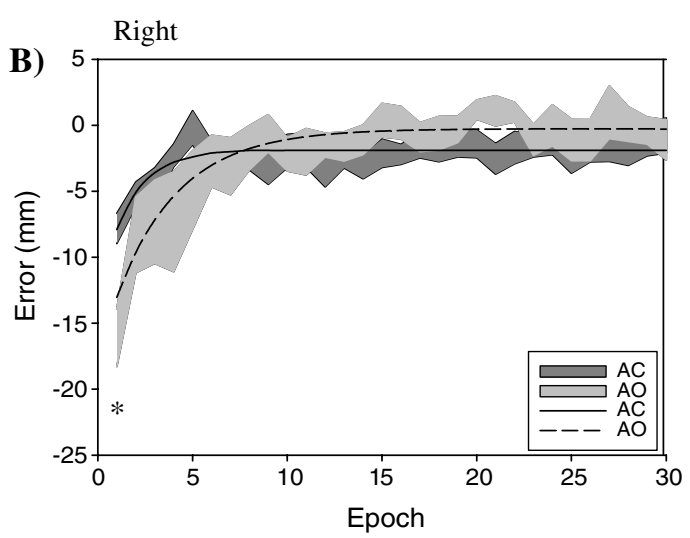

performance. The lines indicate mean exponential fit while the shaded areas indicate final position error \pm S.E. across subjects. Each epoch contained three trials $(* P \leq 0.038)$ 


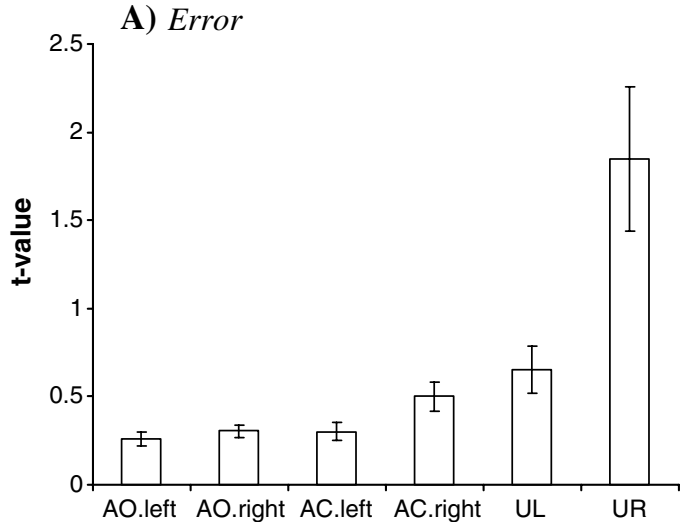

Fig. 6 Adaptation rates, $\tau$, extracted by curve fitting of group average epoch data. All values have been inverted. a For error data the unimanual conditions (UL, UR) have larger $\tau$ values in comparison to the alternating conditions. This suggests a faster adaptation rate in the unimanual conditions. The right hand

alternation of vision was a factor in the slowed adaptation rate then control 2 would show significant differences to condition UR. A main effect of epoch was found $(F(29,522)=10.883, P \leq 0.0005)$, however the main effect of condition and the interaction between epoch and condition were both non-significant. The learning models applied (Eq. 1) suggest that condition UR adapted at a faster rate $(\tau=1.85$, S.E. 0.41$)$ in comparison to control $2(\tau=1.21$, S.E. 0.181$)$. However, Fig. 7 shows that for both control conditions this difference in adaptation rate was not substantial enough to produce any clear separation of the learning curves.

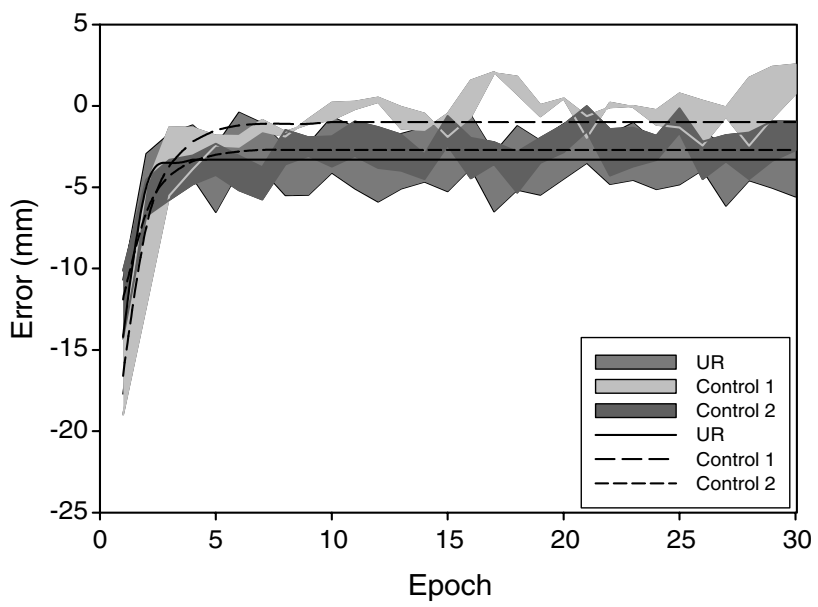

Fig. 7 A comparison of the control conditions with condition UR. Similar performance is seen across conditions however the $\tau$ values suggest that the UR condition adapted at a faster rate. This can be seen as the line indicating the mean exponential fit for the UR condition reaches the plateau level of performance at earlier epoch in comparison to the control conditions. The lines indicate mean exponential fit while the shaded areas indicate final position error $\pm \mathrm{SE}$ across subjects

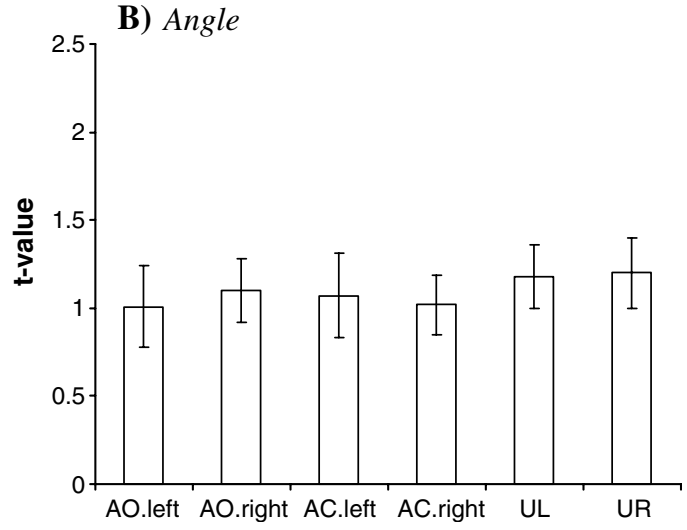

within the AC condition has a larger $\tau$ value than the right hand within condition AO, indicating a faster adaptation rate. b For angle data similar $\tau$ values suggest comparable performance across conditions and hands (error bars indicate standard error of the $\tau$ estimates)

\section{Opposite eye exposure}

To ensure these results were not due to a specific relationship between eye and hand another group were tested. This group was tested with the same conditions as the AO group; however the opposing eye was used. For example when the right hand moved, vision was displaced rightwards but using the left eye and viceversa. Five subjects participated in this condition (three females, two males, mean age $23 \pm 9.7)$. Two mixed ANOVAs [conditions $(2) \times$ epochs (30)] were used to compare it with the original AO condition. For both the left and right hands, there were no significant differences suggesting the eye used was not important. The $\tau$ values (left hand-0.3 \pm 0.098 , right hand $-0.34 \pm 0.82$ ) were found to be similar to those of condition AO (left hand-0.26 \pm 0.4 , right hand $-0.31 \pm 0.034$ ) supporting the claim that there were no significant differences between these conditions.

These results suggest that the slowed adaptation rate within the alternating conditions (AO and $\mathrm{AC}$ ) was not solely due to the task involving alternating movement and vision. In support of this view the $\tau$ values (Fig. 6a) within the alternating conditions were at least $50 \%$ smaller in comparison to the values within the two control conditions. This suggests that factors other than the specifics of the task were influencing the rate of adaptation.

\section{Post-adaptation}

For the post-adaptation session one sample $t$-tests compared the performance of each condition within 
epochs 1 and 5 against a value of 0 . As the data had been previously adjusted for baseline performance, this tested for a return to this baseline value. For all conditions there was a significant difference between epochs 1 and $0(t=3.285, d f=9, P \leq 0.009)$. This suggests that when the prismatic displacement was removed an aftereffect error in the opposite direction occurred. This error is observable for both the angle and error data (Figs. 2,3). The only group which had not returned to baseline performance by epoch 5 was the right hand within condition AC (error data), where a significant difference was still found $(t=3.290$, $d f=9, P=0.009)$. It is apparent that for all conditions, but in particular the unimanual conditions, the aftereffect has a greater initial error in comparison to the corresponding initial displacement caused by the prisms. An exponential learning model was applied to the average post-adaptation data; however due to the limited number of trials within this session reliable parameters could not be fit.

Within the unimanual conditions (UL, UR and control 2) the pre- and post-adaptation sessions involved bimanual movement and so tested for aftereffects in the other hand. T-tests compared epoch 1 within the post-adaptation session with epoch 5 of the pre-adaptation session. No significant effects were found, suggesting that there were no differences between pre- and post-adaptation for the non-adapted hand. Thus the non-adapted hands did not show any significant aftereffects.

\section{Discussion}

The present study aimed to test if the left and right hands could independently adapt to prismatic visual displacements during an alternating pointing task. Within one condition the subjects learnt to adapt to visually opposing (AO) and in another visually consistent (AC) displacements. These alternating conditions were compared to unimanual conditions (UL and UR). There were two measures of task performance; hand-path direction error at peak tangential hand velocity (angle) and the final position error of the index fingers for each pointing movement towards the distal target (error). It was thought that the angle measurement would represent the pre-planned aspect of the movement, whilst the error measurement would also reflect online correction.

Bock et al. (2005) found using a performance measure similar to the angle parameter, that the two limbs were able to adapt to opposing visual displacements concurrently without negative interference. Our results also show clear evidence of adaptation to the opposing visual displacements within the AO condition. When using the angle movement parameter we found no significant differences between the two hands in their initial displacement, learning rate or final level of adaptation. As opposing aftereffects were also found for both hands, we assume that the two adaptive states reflecting changes in sensory-motor translation were adopted and thus are indicative of motor learning (Bock et al. 2005).

In contrast to Bock et al.'s (2005) results we found that within the error data the unimanual conditions have significantly faster adaptation rates in comparison to the alternating conditions. This is based on significant interactions between conditions and epochs and larger $\tau$ values for the unimanual conditions. Hence, subjects in the unimanual conditions reach the plateau stage of adaptation in fewer movements. The plateau indicates the asymptotic portion of the curve where increasing experience with the visual perturbation no longer leads to substantial improvements in performance (Wang and Sainburg 2004). This suggests that during the alternating task negative interference occurred between the limbs resulting in a slowed adaptation rate.

It has been shown that if a subject is simultaneously exposed to two opposing displacements, convergence occurs which is suggestive of neural cross-talk (Craske and Crawshaw 1974). The present set-up of alternating exposure appears to have allowed the brain to dissociate the two adaptive states. However, the continuous shifting of attention from not only one arm to the other but from one displacement to the other could produce a performance decrement (Jackson et al. 1999). Control 1 involved an alternating movement where only the right limb was exposed to a visual displacement. It was hypothesized that if the alternation of the movement was causing the slowed adaptation rate then the right hand performance would show significant differences to the right hand within the unimanual condition (UR). The $\tau$ values suggest that the alternation did indeed result in a slower adaptation rate. However, as these reduced $\tau$ values were still at least double the values found within the alternating conditions ( $\mathrm{AO}$ and $\mathrm{AC}$ ), and as no significant interaction was found [Condition (control 1 vs UR) vs Epoch] it implies that the continuous shifting from one limb to another was not a pivotal factor in causing the observed interference.

The unimanual and alternating conditions not only differed by the numbers of arms used but also by the number of eyes used. Control 2 involved a unimanual movement where unilateral vision alternated after 
every movement. It was hypothesized that if the slowing of the adaptation rate was due to the subjects having to switch between left and right eye vision then a significant difference would be found between this and the unimanual right (UR) condition. The $\tau$ values again suggest that the alternation of vision does influence the adaptation rate. However, the lack of an interaction between conditions and epochs and the substantially larger $\tau$ values in comparison to the alternating conditions indicate that even though computational resources may have been partially engaged in dealing with the alternating vision this alone fails to explain the larger reduction in adaptation rate occurring in the alternating conditions.

The inconsistent results from the angle and error data suggest that interference did not occur during the feedforward aspect of movement. As the differences between alternating and unimanual conditions were only in the error data, we propose that interference between the limbs occurred within the online correction stage of movement. Note that with one exception the adaptation rates for the error data are lower than those for the angle data suggesting that negative interference affected online correction but not feedforward commands. Servos and Goodale (1994) believe visual information stored in extrinsic coordinates is essential for the online control of visually guided movements. The prismatic displacement involved in the present task directly affects this component of online correction. If it is accepted that the limbs are controlled independently (Bock et al. 2005) it is therefore pivotal that the displacements are dissociated in order for each limb to adapt to the respective displacement and thus be able to use vision accurately during online correction. Interference and a slowed adaptation rate may occur as a result of this requirement to dissociate the visual information from the alternating displacements. Control 2 indicates that this dissociation is not coupled with the alternation of vision; this dissociation seems only relevant during an alternating task involving two limbs. As this interference also occurred within the AC condition, it implies that it may be an implicit consequence of using the left and right limb independently. It is already known that this dissociation does not occur during unimanual movement where external contextual cues are required to learn opposing adaptive states (Osu et al. 2004; Wada et al. 2003).

The slowed adaptation rate was found to occur within both alternating conditions. However there does seem to be a grading effect where a greater amount of interference occurred within the $\mathrm{AO}$ condition than in the $\mathrm{AC}$ condition. This view is based on the significant interactions found between conditions ( $\mathrm{AO}$ vs $\mathrm{AC}$ ) and epochs, the significant difference between the conditions at epoch 1 for the right hand, and the larger $\tau$ value for the right hand within condition AC. These results suggest that for the right hand in particular a smaller amount of negative interference occurred within the AC condition. Bays et al. (2005) suggest that the ability to retain motor memories for different tasks depends on the degree to which their representations conflict in working memory. We propose that the AC condition involved a smaller amount of interference. As the interference was seen within online correction, the conflict may be the perceived distance between the targets. For the AC condition the prismatic displacements would have not changed this distance; however within the $\mathrm{AO}$ condition the perceived distance would have increased from 32 to $46 \mathrm{~cm}$ as a result of the $11.4^{\circ}$ opposing displacements. If interference between tasks is due to the degree to which their representations conflict (Bays et al. 2005; Bock 2003) and if visual information stored in extrinsic coordinates is essential for the online control of visually guided movements (Servos and Goodale 1994) then this increased distance between the targets may result in the greater interference seen within the AO condition.

Bock et al. (2005) suggest that the interaction between the adaptive states during the alternating tasks is functional, with the use of a given arm acting as a cue to switch between two available states. If a single adaptive area of the brain is used for both hands, this would have to functionally adjust in order to prevent interference. Within unimanual literature there is a dispute as to whether two opposing environments can be learnt by one limb if they are randomly or continually changing. Karniel and Mussa-Ivaldi (2002) exposed subjects to two alternating dynamic forcefields and found even after 4 days of learning that they were unable to form two independent internal models. They concluded that the central nervous system employed a single internal model when dealing with a sequence of perturbations. In contrast Osu et al. (2004) and Wada et al. (2003) have shown that if the two force-fields are paired with contextual cues the formation of two internal models can take place. However, this learning is significantly slowed in comparison to learning to adapt to one force-field. Wada et al. (2003) conclude that the key determinants of multiple internal model learning are the relative efficiency of contextual information and the similarity of the two environments. With regard to the current experiment it seems likely that using separate limbs was a very efficient cue in selecting the correct feedforward movement command. This is shown by there being no 
significant differences between the alternating and unimanual conditions within the angle data which is thought to represent the feedforward component of the task.

We observed negative aftereffects within the postadaptation session. Bimanual coupling is characterized by two independent movements converging (Franz and Ramachandran 1998). The two hands produced smaller aftereffects in AO compared to the unimanual conditions, suggesting they converged as a result of the two hands moving simultaneously. The AC condition produced slightly larger aftereffects than the unimanual conditions. This can also be explained through bimanual coupling as the two limbs moved in the same anti-clockwise direction forcing each limb further in that direction.

An interesting finding is that generally all the aftereffects had greater initial amplitude, in comparison to the error caused by the onset of displacement. This is surprising as they are presumably manifestations of a single underlying change (Harris 1965). However, this may be simply due to the postadaptation session only involving vision of the movement end point and therefore not having any online feedback. This would cause the error to be larger in comparison to full vision as in the prism adaptation session. Note that the average error in the baseline condition, with terminal feedback, was larger than the final error in the adaptation session, thus supporting this suggestion. Finally, the pre- and post-adaptation sessions involved simultaneous hand movement in all groups, whereas the adaptation session involved either unimanual or alternating action. This also may contribute to the difference in adaptation and aftereffect errors.

As the post-adaptation session always involved a bimanual movement, bimanual coupling could have resulted in a bimanual aftereffect following the unimanual conditions. However, for the non-adapted hands there were no significant differences between the pre- and post-adaptation error values within both unimanual conditions. This suggests that adaptation of one hand to the visual displacement did not interfere with the subsequent performance of the non-adapted hand and that bimanual coupling was specific to the alternating conditions. This specificity suggests that the coupling seen in the alternating condition is engaged by the adaptation process; if only one hand is adapted, the plastic process is constrained to affect only that hand.

In summary this present paper supports the view that two adaptive states can be learnt concurrently during an alternating bimanual task. The slowed adaptation rate within the alternating conditions is believed to be due to negative interference between the two limb controllers during online correction. Two control conditions suggest that the intermittent nature of the task and the alternation between the left and right eye are factors which influence the adaptation rate but do not fully explain the negative interference. It seems likely that during alternate movement, use of each limb provides contextual cues to switch between the relevant internal models. We propose that the negative interference may result from the requirement to dissociate the visual information needed for online correction of each limb.

\section{References}

Bays PM, Flanagan JR, Wolpert DM (2005) Interference between velocity-dependent and position-dependent forcefields indicates that tasks depending on different kinematic parameters compete for motor working memory. Exp Brain Res 163:400-405

Bock O (2003) Sensorimotor adaptation to visual distortions with different kinematic coupling. Exp Brain Res 151:557560

Bock O, Worringham C, Thomas M (2005) Concurrent adaptations of left and right arms to opposite visual distortions. Exp Brain Res 162:513-519

Craske B, Crawshaw M (1974) Adaptive changes of opposite sign in the oculomotor systems of the two eyes. Q J Exp Psychol 26:106-113

Eliassen JC, Baynes K, Gazzaniga MS (2000) Anterior and posterior callosal contributions to simultaneous bimanual movements of the hands and fingers. Brain 123:2501-2511

Franz EA, Ramachandran VS (1998) Bimanual coupling in amputees with phantom limbs. Nat Neurosci 1:443-444

Harris CS (1965) Perceptual adaptation to inverted, reversed, and displaced vision. Psychol Rev 72:419-444

Jackson GM, Jackson SR, Kritikos A (1999) Attention for action: coordinating bimanual reach-to-grasp movements. Br J Psychol 90:247-270

Johansson RS, Theorin A, Westling G, Andersson M, Ohki Y, Nyberg L (2006) How a lateralized brain supports symmetrical bimanual tasks. PLoS Biol 4:1-10

Karniel A, Mussa-Ivaldi FA (2002) Does the motor control system use multiple models and context switching to cope with a variable environment? Exp Brain Res 143:520-524

Mikaelian H, Malatesta V (1974) Specialized adaptation to displaced vision. Perception 3:135-139

Oldfield RC (1971) The assessment and analysis of handeness: the Edinburgh inventory. Neuropsychologia 9:97-113

Osu R, Hirai S, Yoshioka T, Kawato M (2004) Random presentation enables subjects to adapt to two opposing forces on the hand (vol 7,pg 111, 2004). Nat Neurosci 7:314

Prablanc C, Tzavaras A, Jeannerod M (1975) Adaptation of the two arms to opposite prism displacements. Q J Exp Psychol 27:667-671

Servos P, Goodale MA (1994) Binocular vision and the online control of human prehension. Exp Brain Res 98:119-127

Swinnen SP, Wenderoth N (2004) Two hands, one brain: cognitive neuroscience of bimanual skill. Trends Cogn Sci $8: 18-25$ 
Wada Y, Kawabata Y, Kotosaka S, Yamamoto K, Kitazawa S, Kawato M (2003) Acquisition and contextual switching of multiple internal models for different viscous force fields. Neurosci Res 46:319-331

Wang JS, Sainburg RL (2003) Mechanisms underlying interlimb transfer of visuomotor rotations. Exp Brain Res 149:520-526

Wang JS, Sainburg RL (2004) Interlimb transfer of novel inertial dynamics is asymmetrical. J Neurophysiol 92:349-360
Wenderoth N, Puttemans V, Swinnen SP (2003) Bimanual training reduces spatial interference. J Mot Behav 35:296308

Wenderoth N, Debaere F, Sunaert S, Van Hecke P, Swinnen SP (2004) Parieto-premotor areas mediate directional interference during bimanual movements. Cereb Cortex 14:11531163 